\title{
1 Learning cell communication from spatial graphs of cells
}

2 David S. Fischer ${ }^{1,2, *}$, Anna C. Schaar ${ }^{1,3, *}$, Fabian J. Theis ${ }^{1,2,3,+}$

$3{ }^{1}$ Institute of Computational Biology, Helmholtz Zentrum München, 85764 Neuherberg, Germany

4 'TUM School of Life Sciences Weihenstephan, Technical University of Munich, 85354 Freising, Germany

5 BDepartment of Mathematics, Technical University of Munich, 85748 Garching bei München, Germany

6 + correspondence to fabian.theis@helmholtz-muenchen.de

7 * These authors contributed equally

Tissue niches are sources of cellular variation and key to understanding both single-cell and tissue phenotypes. The interaction of a cell with its niche can be described through cell communication events. These events cannot be directly observed in molecular profiling assays of single cells and have to be inferred. However, computational models of cell communication and variance attribution defined on data from dissociated tissues suffer from multiple limitations with respect to their ability to define and to identify communication events. We address these limitations using spatial molecular profiling data with node-centric expression modeling (NCEM), a computational method based on graph neural networks which reconciles variance attribution and communication modeling in a single model of tissue niches. We use these models in varying complexity across spatial assays, such as immunohistochemistry and MERFISH, and biological systems to demonstrate that the statistical cell-cell dependencies discovered by NCEM are plausible signatures of known molecular processes underlying cell communication. We identify principles of tissue organisation as cell communication events across multiple datasets using interpretation mechanisms. In the primary motor cortex, we found gene expression variation that is due to niche composition variation across cortical depth. Using the same approach, we also identified niche-dependent cell state variation in CD8 T cells from inflamed colon and colorectal cancer. Finally, we show that NCEMs can be extended to mixed models of explicit cell communication events and latent intrinsic sources of variation in conditional variational autoencoders to yield holistic models of cellular variation in spatial molecular profiling data. Altogether, this graphical model of cellular niches is a step towards understanding emergent tissue phenotypes.

Cells interact on multiple length-scales through direct contact of surface-bound receptors and ligands, tight-junctions and mechanical effects, as well through indirect mechanisms, including soluble factors and vesicles $^{1}$. These communication events can usually not be directly observed but are critical to understand emergent phenomena in tissue niches. However, the sender and receiver cell of a communication event are often characterized by molecular signatures, both facilitating the signalling, such as ligand and receptor expression ${ }^{2-4}$, and resulting from the signalling event, such as intracellular cascades induced by receptor activation. These molecular signatures are used in computational methods to infer latent cell communication events in a tissue. Core assumptions inherent in these algorithms can be summarized in two groups: First, the co-occurrence of ligand and receptor expression across cell-types is used in multiple computational models to suggest putative axes of cell communication ${ }^{2,3}$. Second, from a statistical point of view, cell communication is a source of cellular variance. The gene expression of a receiving cell depends on the sending cells in the local tissue niche, thus inducing a statistical dependency that can be used to infer communication events ${ }^{2,5}$. Here, we address three core issues inherent in cell communication inference methods founded on these two assumptions using spatial information. First, axes of cell communication cannot be necessarily disentangled based on data from dissociated cells because of the large number of potential interactions in a population of cells. We propose to use spatial information from image-structured molecular profiling assays to constrain the inference of such gene expression dependencies between neighboring cells. For this purpose, we define a prior distribution on the possibility of a communication event between two cells as a function of their distance, using space to prioritize pairs of cells for which communication is molecularly plausible. Second, cell pairs with matching ligand and receptor expression can occur in a tissue even in the absence of communication, for example because of spatial separation in the tissue architecture. We propose to use the receiver cell molecular signature as conclusive evidence for the presence of a communication event. Third, molecular signatures of receiver cells can be observed independent of ligand-receptor quantification. Therefore, cell communication inference should be possible both in the absence of a reliable quantification of ligand or receptor genes, which occurs in targeted molecular profiling assays, or in cases in which these genes are expressed at low levels, and in receptor protein-free communication, such as in physical interactions. We define a generalized framework to detect receiver cell signatures detached from ligand-receptor definitions. 
bioRxiv preprint doi: https://doi.org/10.1101/2021.07.11.451750; this version posted July 12, 2021. The copyright holder for this preprint (which was not certified by peer review) is the author/funder, who has granted bioRxiv a license to display the preprint in perpetuity. It is made available under aCC-BY 4.0 International license.

57 We reconcile these assumptions in a statistical model for cell communication events in spatial molecular profiling

58 data, referred to as a node-centric expression model (NCEM). NCEMs are trained on segmented

59 image-structured molecular data from assays with subcellular resolution, such as immunohistochemistry ${ }^{6,7}$,

60 imaging mass cytometry ${ }^{8}$, and MERFISH $^{9}$ (Fig. 1a). We enforced a neighborhood constraint on communication

61 events using spatial graphs of cells, where the graph serves as prior for cellular communication. The resulting

62 model is a graph neural network ${ }^{10,11}$ and constitutes an extension of models of dissociation-based data, in which

63 cells are modeled independently, to a spatially-constrained model of cell-cell dependencies (Fig. 1a). This model

64 relies on the stratification of cells into clusters that can then be treated as categorical sender and receiver

65 cell-types in communication events. Importantly, the directionality of these sender-receiver signalling is defined

66 on the level of pairs of single cells by the statistical association of the molecular state of the receiver on the

67 sender. We discovered these statistical associations in a model of the molecular state of cells conditioned on the

68 putative sender cell-types present in their respective spatial neighborhoods. The complexity of the spatial

69 dependencies observed in a tissue strongly depends on the tissue architecture and the ability of the molecular

70 readout to capture the signatures of these dependendencies. Therefore, the cell communication model

71 complexity needs to be adapted to the scenario at hand. We studied three increasingly complex models of

72 cell-cell dependencies. First, we propose a linear graph neural network that can be framed as a graph-aware

73 generalized linear model (GLM) to model linear expression effects of cell communication. Secondly, we

74 generalize NCEMs to nonlinear graph neural networks that can account for higher-order cell interactions. Third,

75 we consider generative latent variable models that also model confounding latent sources of variation, such as

76 cell intrinsic effects. We interpret NCEM fits in terms of cell communication patterns. We demonstrate cell

77 communication inference with NCEMs on five datasets (Online methods, Supp. Figure 2): a MERFISH dataset of

78 mouse brain ${ }^{12}$ (MERFISH - brain) of 634 images across with 254 genes observed in 284,098 cells, a chip

79 cytometry dataset of an inflamed colon (Data Availability) (chip cytometry - colon) of two images with 19 genes

80 observed in 11,321 cells, a MIBI TOF dataset of colorectal carcinoma ${ }^{8}$ (MIBI TOF - cancer) of 58 images with 36

81 genes observed in 63,747 cells, a MELC dataset of tonsils ${ }^{7}$ (MELC - tonsils) of one image with 51 genes

82 observed in 6,991 cells and a CODEX dataset of colorectal cancer ${ }^{13}$ (CODEX - cancer) of 140 images with 57

83 genes observed in 272,266 cells. We discover cell-cell dependencies at molecularly plausible length scales and

84 attribute molecular variation within cell-types to niche composition. The inferred interactions between cells serve

85 as a powerful mechanism to interpret niche composition variation and its effect on the contained cell.

\section{Results}

\section{Node-centric expression models describe cell-cell dependencies on spatial graphs}

88 We infer cell communication from a cell-type-specific gene expression signature that can be predicted from

89 cell-types in the spatial neighborhood. The data type discussed here consists of image-structured data from

90 molecular profiling assays of RNA or proteins, where pixels correspond to tissue slice positions. Each channel

91 contains a molecular abundance readout of a specific gene. To prepare this analysis, we first used segmentation

92 masks to assign pixels in image-structured data to cells (Fig. 1a,c). We then extracted the mean gene expression

93 from these segments to build cell-wise gene expression vectors and clustered these molecular vectors to assign

94 cells to discrete cell-types (Fig. 1a,c). Based on the cell segmentation, we defined the niche of a cell as the set of

95 cells within a circle centred on the cell's center. The radius of this circle, the "resolution", is a model

96 hyperparameter (Online Methods, Fig. 1a). We define a node-centric expression model (NCEM) as a function

97 that maps a cell's type and its spatial context to the cell's observed gene expression vector (Fig. 1b). This

98 function is a graph neural network in which the node labels are the cell-wise gene expression vectors ${ }^{10}$. Below,

99 we discuss both multilayer graph neural networks and a single-layer model with an indicator graph aggregator

100 function which is equivalent to a linear model (Fig. 1b, Online Methods): The indicator function aggregator

101 summarises a set of one-hot encoded cell-types in a niche to a vector with binary elements that indicate the

102 presence of each cell-type in the niche. This binary embedding is parameter-free and a transformed covariate

103 space with fixed dimensions and thus suitable for a linear model. In addition to this indicator embedding, we also

104 use parametric neighborhood-embeddings for nonlinear NCEMs (Online Methods). The model of a gene

105 expression vector conditioned on a cell-type reflects the assumption that niches modify gene expression states of

106 cell-types, but do not cause cell-type conversions themselves. This conditional model also reflects the

107 assumption that much spatial information is contained in the gene expression profile of a single cell ${ }^{14}$ and that

108 spatial covariates are primarily useful to supervise variance decomposition of gene expression vectors. In

109 summary, the input data to NCEMs consists of three groups: the set of input cell-type labels, the output gene

110 expression features and the spatial proximity graph. 
bioRxiv preprint doi: https://doi.org/10.1101/2021.07.11.451750; this version posted July 12, 2021. The copyright holder for this preprint (which was not certified by peer review) is the author/funder, who has granted bioRxiv a license to display the preprint in perpetuity. It is made available under aCC-BY 4.0 International license.

112 The input cell-type labels group cells to both sender and receiver cell-types, this annotation can be more or less

113 coarse according to the cell-type clustering resolution. Cell-types from one sender class emit a common signal,

114 and cell-types of one receiver class share a common response gene expression signature for a given signal. The

115 coarseness of the sender and receiver cell-type classes serves as an inductive prior which regularizes the model

116 by reducing the number of parameters that model cell communication. For example, an overly fine-grained set of

117 receiver cell-types could attribute niche-dependent cellular variation within a cell-type to between-cluster

118 variation, thus failing to attribute this axis of variation to niche composition.

119

120

The identity and number of molecular species measured in a spatial molecular profiling assay vary strongly across protocols and studies. Accordingly, the observable neighborhood-induced gene expression effects vary equally strong. One could include further cell-features related to morphology and molecule distribution in the cell state $^{15}$ to improve the description of the molecular state of a cell to subcellular gene expression variation.

In a spatial proximity graph, cells are connected by edges if their segment centers are not further apart than the resolution of the model ${ }^{16}$ (Fig. 1a). The choice of this resolution depends on the modeled molecular mechanisms of communication, which vary strongly between contact-based and paracrine communication, for example. In this study, we chose multiple resolutions for each dataset in separate analyses. Each resolution is associated with a separate graph that has a specific node degree distribution which depends on the overall tissue architecture. We defined the distances for each dataset such that they cover the range of average node degrees of the given dataset (Supp. Fig. 1b). This screening of resolutions is useful for discovery of statistical dependencies. On the other hand, a fixed, single resolution can be used to test specific hypotheses of cell communication events on a length scale defined by prior knowledge based on molecular mechanisms.

\section{Linear NCEM identify cell communication on consistent spatial length scales}

We model neighborhood-induced cell state changes with a linear graph neural network that predicts the state of a receiver cell conditioned on the presence of putative sender cell-types present in its neighborhood. This model is equivalent in architecture to a graph-aware GLM (Online Methods) and, therefore, represents a convex optimization problem. While limited in their ability to model higher-order cell interactions, such linear models can be readily interpreted in terms of effect sizes, can be used by non-specialists because of their favourable optimization characteristics and allow for correction for confounding factors such as biological conditions.

First, we established the presence of intra-cell-type variance in a variance decomposition on all five datasets (average intra-cell-type variance $40.6 \%$ ), even in those datasets with very few genes assayed (Supp. Fig. 2, Online Methods). We fit Gaussian GLMs on transformed single-cell expression measurements across multiple neighborhood sizes with four term groups: image covariates, the cell-type, the presence of putative sender cell-types in the neighborhood (the niche), and an interaction term between the cell-type and the niche (Online Methods). As a null model, we included empty neighborhoods and neighborhoods larger than the considered images. We found that these GLMs were most predictive on an intermediate-length scale of around $69 \mu \mathrm{m}$ in all five datasets (Fig. 1d), showing that cell-cell dependencies indeed only appear on length scales characteristic of direct and short-range molecular mechanisms of cell communication. With further increasing neighborhood sizes, the prediction performance drops again to the level of nonspatial models, indicating that the spatial effect is not simply due to overfitting to images. The NCEMs outperformed non-spatial baseline models by an average $\Delta R^{2}$ of 0.0175 . This difference was significant in paired t-test between baseline model and best spatial model of $(p<$ 0.05 ) across five datasets (Fig. 1d). The $\Delta R^{2}$ is small compared to the baseline model $R^{2}$ that characterizes between-cell-type variance $(0.39-0.79)$ because the cell-type information accounts for a large fraction of the transcriptomic variance in these samples. Between-cell-type variance often dominates variance in single-cell transcriptomics which is a phenomenon that allows for cell-type assignment through clustering algorithms, for example $^{17-19}$. The remaining within-cell-type variance decomposes into technical noise and within-cell-type biological variance. The biological within-cell-type variance can be decomposed into spatial and non-spatial effects. Here, we only model the spatial component, thus explaining the low magnitude of explained within-cell-type variance compared to the total within-cell-type variance. The increased $R^{2}$ achieved by NCEM can be attributed to the niche composition information inherent in the model, thus providing an algorithmic handle on niche biology.

In addition, we also fit GLMs with fewer parameters, without cell-type to niche interaction terms, keeping only non-receiver-specific sender effects, which are less susceptible to overfitting, and also found dependencies on 
bioRxiv preprint doi: https://doi.org/10.1101/2021.07.11.451750; this version posted July 12, 2021. The copyright holder for this preprint (which was not certified by peer review) is the author/funder, who has granted bioRxiv a license to display the preprint in perpetuity. It is made available under aCC-BY 4.0 International license.

167 similar length scales (Supp. Fig. 3, Online Methods). We hypothesized that different molecular mediators of

168 cell-cell dependency on different length-scales apply to different cell-types. Indeed, the model performance as a

169 function of length scale by target cell-type varies between cell-types (Supp. Fig. 4).

170

\section{Niche composition explains cellular variation}

171 Next, we interpreted the effects learned by the linear NCEMs. To identify neighborhood-induced molecular states

172 in the primary motor cortex, we selected L2/3 IT cells from the MERFISH - brain dataset ${ }^{12}$ based on their strong

173 spatial effects on cell state (Supp. Fig. 4) and their large abundance, with 41,996 cells across the dataset (Supp.

174 Fig. 1a). To identify neighborhood-induced effects on the molecular states without confounding the analysis by

175 between-image batch and condition effects, we clustered and embedded L2/3 IT cells from a single image based

176 on the molecular information alone (Fig. 2a, Online methods). Indeed, the relative prediction performance of the

177 graph-aware GLM at the optimal resolution compared to nonspatial GLM varies across these clusters (Fig. 2b),

178 as measured by the difference of their respective $R^{2}$ on individual cells. Using the presence of different cell-types

179 in the neighborhood as a binary labels vector, we performed a cluster enrichment analysis with Fisher's exact test

180 on this clustering (Fig. 2c,d, Online Methods) to annotate clusters with enriched neighborhoods: sub cluster 0 is

181 associated with Scng and VLMC, and cluster 4 with L4/5 IT cells, for example (Fig. 2d). We ordered cell-types as

182 putative sender types of L2/3 IT cells by their maximal cluster-wise enrichment $p$-value. This ordering of

183 cell-types is very different from the ordering obtained from contact frequencies and CellphoneDB ${ }^{3}$ analysis on this

184 image (Kendall rank correlation of -0.04 and -0.12 , p-value of 0.79 and 0.45 , respectively, Supp. Fig. $6 a$, $7 b$,

185 Online methods), highlighting the novel quality of information captured by this approach. Depending on the

186 analysis setting, either L4/5 IT cells and Scng or L4/5 IT cells and VLMC are also discovered as putative partners

187 of L2/3 IT cells by CellphoneDB (Supp. Fig. 7a,b), showing that core associations are reproduced. Next, we

188 established that the L2/3 IT cell sub clusters are spatially localised in distinct areas of the primary motor cortex ${ }^{12}$.

189 Sub cluster 0 is closer to the layer of Scng and VLMC, whereas sub cluster 4 is closer to the layer of L4/5 IT cells

190 (Fig. 2c,d). This spatial patterning becomes apparent when the sub-cluster-wise relative performance spatial and

191 non-spatial model (Fig. 2b) are broken down to cell-wise comparisons and assigned to cells in their spatial

192 context: The spatial model outperforms the non-spatial model strongly on margins of the L2/3 IT layer in the

193 motor cortex, such as in sub cluster 4 of the L2/3 IT cells (Fig. 2e). We repeated this analysis by applying the

194 same models, which were trained on all images, to a second image and found similar associations to

195 neighborhoods, such as significant associations to Scng, VLMC, and L4/5 IT cells (Supp. Fig. 5b-f). In summary,

196 this analysis attributes molecular variation of single cells to their niche and identifies putative sender cell-types

197 which are in line with the overall tissue architecture.

198

199 In an equivalent analysis on the chip cytometry - colon data, we discovered a dependency of CD8 T cells on

200 multiple other cell-types, such as CD8 T cells and lamina propria cells (sub cluster 3) (Supp. Fig. 8a-e),

201 highlighting a dense compartment of T cells in the inflamed colon centred on lamina propria cells (Supp. Fig. 8f).

202 In colorectal cancer, linear NCEM learned a previously established dependency of CD8 T cell states on proximity

203 to the tumor-immune boundary ${ }^{8}$ (Supp. Fig. 9a-e), where sub cluster 1 represents cells close to malignant

204 epithelial cells (Supp. Fig. 9c,f).

206 Next, we considered parameter significance in GLMs as a mechanism to attribute cellular variation to

207 communication events with specific sender cell-types. We used a differential expression test, a Wald test, to test

208 gene-wise cell communication coefficients in the linear model. In the linear model as defined above, the Wald test

209 yields a p-value for the effect of the interaction between each pair of cell-types on each assayed gene in the

210 receiver cell-type. The inference of cell communication with this gene-centric model improves with the number of

211 assayed genes, as only a subset of all genes will display differential expression in response to the

212 communication event. We found receiver cell-type-gene pairs that significantly depend on the neighborhood in

213 the chip cytometry - colon data (Supp. Fig. 10c): Here, one would expect communication events with immune

214 cells because of the inflamed state of the colon. The communication between sender PD1L+ cells and receiver

215 CD8+ T cells was the strongest in terms of the number of differentially expressed genes, suggesting that

216 PD1-mediated T cell exhaustion ${ }^{20}$ plays a role in this tissue. Importantly, this communication is not identifiable

217 from cell contact frequencies (Supp. Fig. 6b) or CellphoneDB analysis (Fig. 7c), as expected for a

218 sender-receiver-specific signature that occurs in a particular tissue niche. Indeed, we found PD1 in the set of

219 genes whose expression is significantly associated with this receiver-sender pair $(p=0.02$ for an absolute change

220 in log-transformed data of -1.92 , Supp. Fig. 10). 
bioRxiv preprint doi: https://doi.org/10.1101/2021.07.11.451750; this version posted July 12, 2021. The copyright holder for this preprint (which was not certified by peer review) is the author/funder, who has granted bioRxiv a license to display the preprint in perpetuity. It is made available under aCC-BY 4.0 International license.

\section{Nonlinear NCEM capture complex cell communication motifs}

222 Linear models for cell communication cannot easily account for higher order interactions between more than two

223 cell-types. Such complex interactions are, however, models of relevant patterns of communication in niches: The

224 communication between two cells $A$ and $B$ may depend on a communication event between $B$ and a cell $C$ which

225 induces ligand expression in B. Moreover, edge properties, such as weights derived from physical distances

226 between cells, or molecular characteristics of communication axes, are difficult to account for in linear models.

227 Here, we discuss multilayer NCEMs which can model both higher order cell communication and edge properties.

228 These graph neural networks can be understood as encoder-decoder models. The encoder is a function of the

229 local context in the graph and yields a latent state that is transformed by a stack of densely-connected layers in

230 the decoder to the predicted expression state of the cell (Fig. 3a). In contrast to autoencoding encoder-decoder

231 models often used to model heterogeneity in single cells from dissociated tissues ${ }^{21,22}$, this encoder-decoder

232 NCEM is a nonlinear regression model that does not receive the expression state in the input (Online Methods).

233

234 First, we established the presence of resolution-dependent prediction performance profiles in encoder-decoder

235 NCEMs in both models with indicator aggregators and graph-convolutional filters, again controlled by empty and

236 large neighborhoods (Fig. 3b). The top-performing length scales were comparable to those obtained on linear

237 models (Fig. 3b). We could identify cell-type-specific communication length scales again (Supp. Fig. 11). Notably,

238 the encoder-decoder models did not outperform linear models on any dataset in terms of reconstruction metrics

239 on test data, which suggests that niche communication is described well by additive pairwise communication

240 events of cells in these tissues at the given sample complexity, rather than higher-order interactions.

242 As any neural network, encoder-decoder NCEMs can be interpreted in terms of cell communication events

243 through gradient analysis. The gradients of output expression values with respect to input receiver cell-type and

244 input sender cell-types approximate the effect that a change in neighborhood composition of a given cell would

245 have on its molecular state (Online Methods). Using this interpretation method, we identified L4/5 IT as a putative

246 sender cell-type for L2/3 IT cells in the MERFISH - brain dataset ${ }^{12}$ (Fig. 3c), which agrees with the strong

247 association between these two cell-types identified in the cluster enrichment (Fig. 2c). Using a similar approach,

248 we identified lamina propria cells as predictors of CD8 T cell state in the chip cytometry - colon dataset (Fig. 3d),

249 reproducing this same association identified with linear NCEM (Supp. Fig. 8c,e).

\section{NCEMs extended to latent cell-intrinsic states explain more variance}

251 Above, we discussed NCEMs as mechanistic models designed to attribute intra-cell-type variance to predictors in

252 the niche. These NCEMs cannot model latent cell states that confound the measured expression data,

253 representing important intrinsic cellular phenomena, such as cell cycle states or differentiation progression. A

254 holistic model of cellular variance needs to account both for these intrinsic and extrinsic effects to disentangle

255 confounded sources of variation ${ }^{5}$. Therefore, we propose an NCEM that accounts for both cell intrinsic latent

256 states and the dependencies of molecular states on the niche. The proposed model is a conditional variational

257 autoencoder (CVAE) in which the condition represents the neighborhood and the cell-type of the cell itself (Fig.

258 4a). In contrast to the node-supervised models discussed above, this CVAE is a node-generative model. This

259 node-generation tasks of learning a distribution over gene expression states conditioned on the cell-type, a local

260 context in the graph, and other covariates, such as chemical perturbation, extends generative models for

261 single-cell gene expression data ${ }^{21,23}$ to spatial cell-cell dependencies.

262

263 Conditional generative models have previously been used to model perturbations of molecular states by small

264 molecules $^{23}$ in the context of data from dissociated tissues. Latent variable models attain much higher predictive

265 performance in reconstruction tasks (Fig. 4b, Supp. Fig. 13a) because they can fit cell-wise states if the model

266 does not suffer from posterior collapse $e^{24}$. However, conditional variational models suffer from a non-identifiability

267 between the variation attributed to the condition and to the latent states because the loss function only constrains

268 the model's marginal likelihood. A CVAE that is ideally suited for style transfer is converged such that the

269 condition-wise marginal posteriors on the latent variables are equal, so that one can sample the full conditional

270 distribution by decoding the latent states observed in a source condition into the target condition. However, there

271 are optima of equal loss values in which the condition information bleeds into the latent variables, resulting in

272 condition-specific latent states that cannot meaningfully be transferred to a target condition. We attempted to

273 address this non-identifiability with aggressive encoder training, an optimization approach capable of favoring

274 convergence to a model that attributes maximal variation in the marginal distribution to the conditional ${ }^{25}$ (Online

275 Methods). We evaluated the ability of a CVAE-NCEM to extrapolate to unseen neighborhoods, a style-transfer

276 setting we call "neighborhood transfer" (Online Methods) using the example of muscular and Lamina Propria cells 
277 from the chip cytometry - colon data (Fig. 4c) and L2/3 IT neurons from the MERFISH - brain dataset ${ }^{12}$ (Supp.

278 Fig. 13b). We did not find consistent neighborhood-transfer performance differences between CVAE-NCEM

279 compared and plain CVAEs, even though peak performance was attained by CVAE-NCEM optimized with

280 aggressive encoder training (Online Methods). This performance analysis suggests that niche states are

281 represented in latent variables, thus confounding resampling in the neighborhood transfer task. Indeed, we could

282 identify multiple significantly enriched neighborhoods in a latent space clustering of the CVAE-NCEM for both

283 examples (Fig. 4d-f, Supp. Fig. 13c-e). This latent variable interpretation shows that the CVAE indeed converges

284 to an optimum that is not desirable in terms of the style transfer task, which can occur because these optimums

285 are not penalized by the CVAE's cost function.

286

287 NCEMs can attribute molecular variation of single-cells to niche composition and thus carry the promise of

\section{Discussion}

293 We presented NCEMs, a graph neural network framework for modeling of cell communication events in spatial

294 omics assays with subcellular resolution, in which cells can be separated in silico through segmentation. The

295 NCEMs presented in this paper are structured in three groups: a linear graph neural network that can be mapped

296 to a GLM, nonlinear graph neural network and CVAEs. We defined the extrapolation to unseen neighborhoods,

297 the prediction of the molecular state of a cell of a given type in a given niche, as a supervision task and proposed

298 an adaptation of this task to generative style transfer models ("neighborhood transfer"). We used NCEMs to

299 discover statistical dependencies between cells on physiologically relevant length scales at a mean of $69 \mu \mathrm{m}$ with

300 a standard error of $14 \mu \mathrm{m}$ across five datasets. We demonstrated that NCEMs could be interpreted based on

301 model parameters in linear models and saliencies in nonlinear models to infer communication events between

302 pairs of cell-types and to disentangle molecular variation in standard cell-centric unsupervised workflows. Using

303 these interpretation mechanisms, we disentangled niche effects in the mouse motor cortex, in the inflamed colon

304 and in colorectal cancer.

305

306 The datasets discussed here are based on technologies with high throughput in the number of cells but relatively

307 few genes measured. For example, imaging-mass cytometry ${ }^{26}$ and CODEX ${ }^{6}$ both profile on the order of tens of

308 genes. Typically, these genes are selected to separate cells from different cell-types, maximizing between-type

309 variance in the data. However, these setups are not designed to profile within-type variance. The more subtle cell

310 states within a cell-type are typically recovered in protocols with large feature spaces, such as spatial single-cell

311 RNA-seq, but also FISH-based protocols, such as seq-FISH ${ }^{27}$ and MERFISH ${ }^{9}$. Although gene expression-based

312 inference of cell communication will probably be improved by techniques that yield larger feature spaces, we

313 could already show in this study that spatial dependencies can be estimated across cross-validation splits of the

314 larger MERFISH dataset with 254 genes measured. We anticipate that such high-dimensional RNA-seq

315 characterizations of single cells in tissues will soon be widely available because of the improvements of seq-FISH

316 and MERFISH protocols and the reduction of spot sizes in spot-based transcriptomics. Datasets with more

317 comprehensive quantification of ligand and receptor expression per cell may allow for further constraints on

318 edges based on the expression of specific ligand and receptor pairs in the participating nodes. Such constraints

319 may further increase interpretability and improve discovery of cell communication events through linear model

320 interpretation and saliency-based analyses. Such constraints may further be derived through imputation of

321 relevant ligand and receptor genes in spatial assays using reference single-cell atlasses ${ }^{14}$. All models proposed

322 here are node-centric and embed a cell locally in the graph, instead of requiring the full graph. Therefore, NCEMs

323 can be used equally well and efficiently on a few large images or on many small images, thereby increasing their

324 relevance for a wider range of scenarios.

326 The datasets modeled here are 2D and do not contain information about cells that are also part of a receiver

327 cell's 3D neighborhood but are located in a different z-section; this limits the capacity of the models shown here

328 to find communication events. One would expect NCEMs to be considerably stronger given 3D data, and we

329 expect such data to become available from serial slicing of tissues, for example.

330

331 Here, we considered graph convolutional networks with linear and indicator graph aggregator functions. Other

332 aggregators are used commonly to compute graph embeddings ${ }^{28}$ and could also be used here. The complexity of 
bioRxiv preprint doi: https://doi.org/10.1101/2021.07.11.451750; this version posted July 12, 2021. The copyright holder for this preprint (which was not certified by peer review) is the author/funder, who has granted bioRxiv a license to display the preprint in perpetuity. It is made available under aCC-BY 4.0 International license.

333 the graph neural network used in the NCEM defines the complexity of the motifs of cell communication that can

334 be discovered. Linear NCEMs discover directional effects between pairs of cells. Higher order effects, that can be

335 captured by deeper graph neural networks, include interactions between different communication events on a

336 target cell and conditional dependencies between communication events, such as in loops. NCEMs do not

337 require complex tissue phenotype labels, which are often unavailable. Effectively, the molecular vectors represent

338 a label on a mid-range length scale of the graph. One can also imagine extending the tissue representation with

339 more global phenotype labels through additional supervision tasks. The coarseness of the input feature space is

340 an important hyperparameter for these graph models. Future work may address the choice of this

341 hyperparameter algorithmically. However, in many cases, there is strong prior knowledge on the assignment of

342 cell states to discrete cell-types.

344 The cell-cell dependencies modeled based on spatial graphs are examples for observational dependencies that

345 violate the common assumption of statistical learning, namely, that single cells are independent and identically

346 distributed. We showed that this assumption could be sacrificed given strong inductive priors, here induced by

347 the spatial graphs, which constrains the modeled communication events from the set of all pairs of cells in a

348 sample to the molecularly plausible ones. Future work could consider further inductive priors which are not

349 necessarily spatial. 


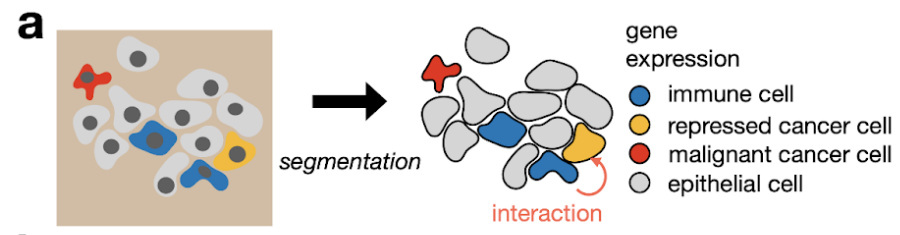

b

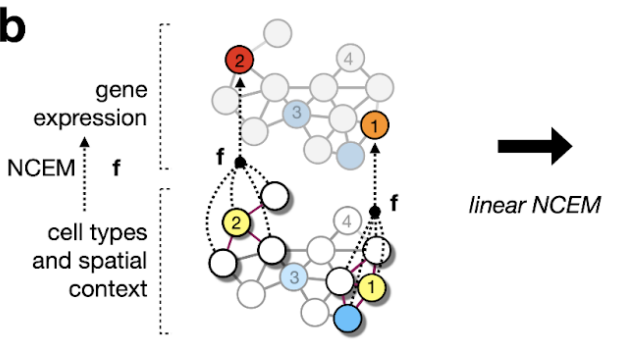

self niche

$\mathbf{f}(1),(O, O, \mathbf{X}))=0$

$\mathbf{f}(2),(0, \mathbf{X}, \mathbf{X}))=0$

$f(3,(O, X, X))=0$

$\mathbf{f}\left(\oplus_{4},(0, \mathbf{X}, \mathbf{X})\right)=0$

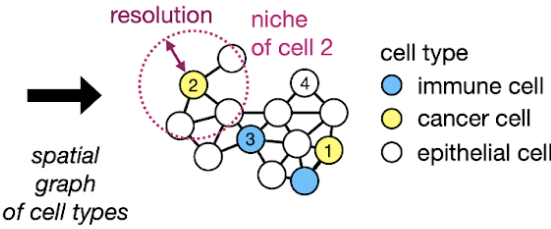

of cell types
C

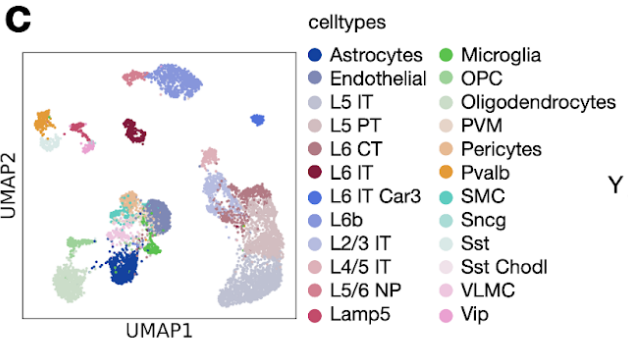

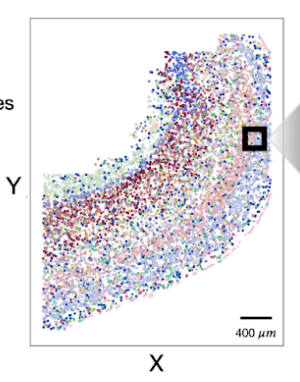
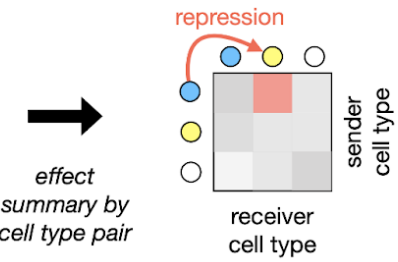
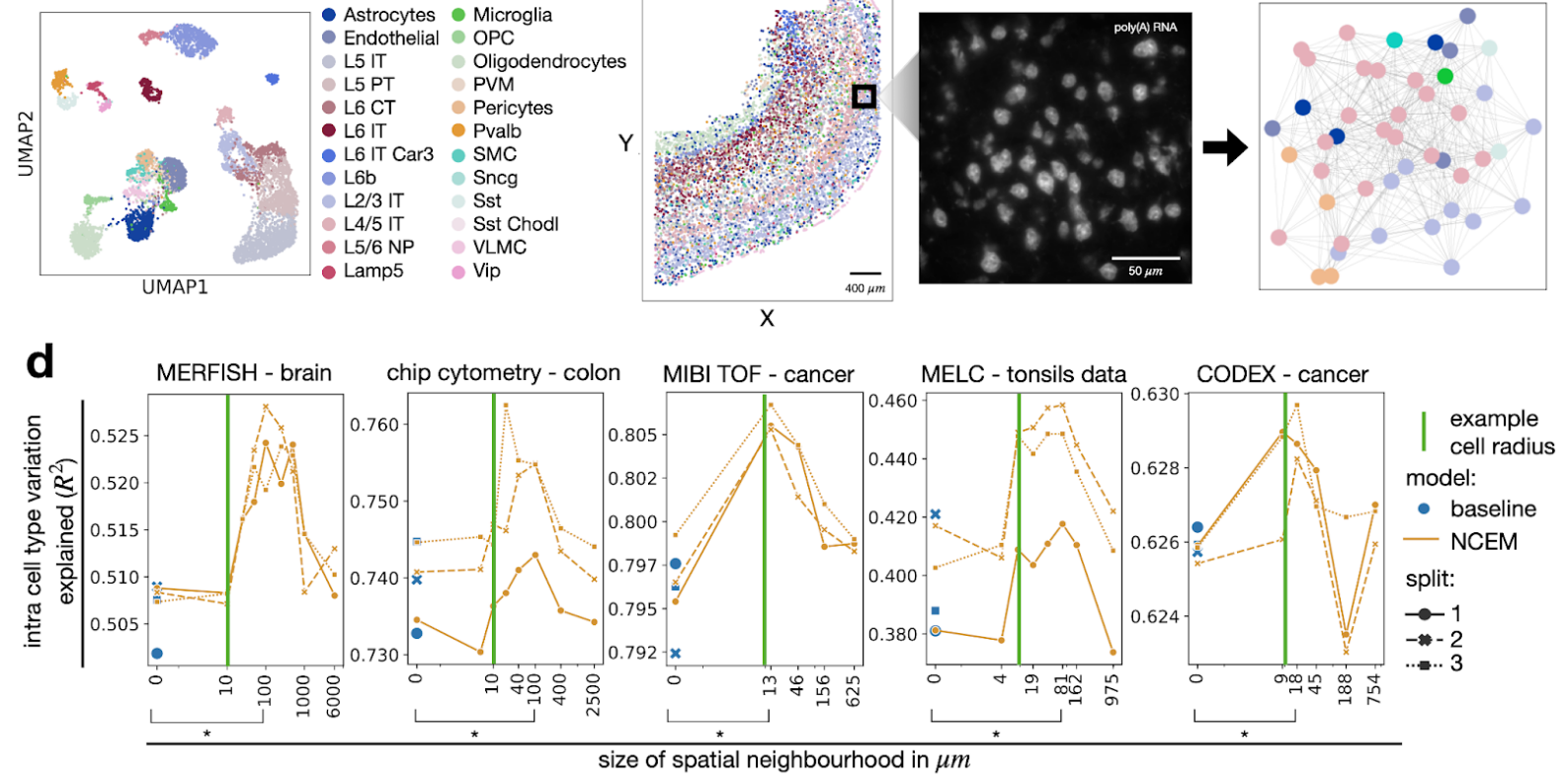

353 Figure 1: Modeling cell communication as spatial cell state dependencies. (a) Spatial graphs of cells can be

354 computed from spatial molecular profiling data. After segmentation of cells or nuclei, each cell is characterized by

355 a vector of gene expression measurements which are coarse-grained in a clustering to categorical cell-types. A

356 graph can be assembled based on proximity of segments. resolution: radius of circle used to define niche. (b)

357 Node-centric expression models (NCEMs) describe the gene expression of a cell (color of the node) as a function

358 of its spatial neighborhood (niche). This function is a graph neural network and can be reduced to a linear model

359 in the simplest case. This linear model contains directional effects from sender to receiver cell-types which can

360 be summarised in an effect matrix between cell-types. Y: gene expression vector of a cell. (c) Proximity graphs in

361 spatial transcriptomics data. Shown are a UMAP of molecular embedding of all cells in slide $153(n=7439$ cells $)$

362 with cell-type superimposed, followed by Slice 153 of mouse brain in the MERFISH - brain dataset with the

363 spatial allocation of all cell-types superimposed, field of view number 486 of the same slice with poly(A) RNA

364 channel superimposed at central z-plane $(z=4.5 \mu \mathrm{m})$, and the spatial proximity graph of the same field of view

365 with a resolution of $100 \mu \mathrm{m}$. (d) Linear models capture neighborhood dependencies in spatially-resolved

366 single-cell data. Shown are the $R^{2}$ values between predicted expression vectors and observed expression

367 vectors for held-out test cells of linear models by resolution in $\mu \mathrm{m}$ with cross validation indicated as point shape

368 and line style with relative outperformance of NCEM model versus baseline model in the MERFISH - brain

369 dataset of $1.77 \%$, in the chip cytometry - colon dataset $1.17 \%$, in the MIBI-TOF - cancer dataset $1.04 \%$, in the

370 MELC - tonsils dataset $4.48 \%$ and in the CODEX - cancer dataset $0.28 \%$. Example cell radius (green line):

371 Example length scale of a cell, here chosen as $10 \mu \mathrm{m}$; baseline (blue dot): a nonspatial linear model of gene

372 expression per cell-type; NCEM: linear model with interaction effects; bracket $\left(^{*}\right)$ : significant difference in paired

373 t-test between baseline model and best spatial model (MERFISH - brain dataset $p=0.030$, chip cytometry - 
bioRxiv preprint doi: https://doi.org/10.1101/2021.07.11.451750; this version posted July $12,2021$. The copyright holder for this preprint (which was not certified by peer review) is the author/funder, who has granted bioRxiv a license to display the preprint in perpetuity. It is made available under aCC-BY 4.0 International license.

374 colon dataset $p=0.019$, MIBI-TOF - cancer dataset $p=0.018$, MELC - tonsils dataset $p=0.029$, CODEX -

375 cancer dataset $p=0.026)$.

376

a

377

$\mathbf{e}$
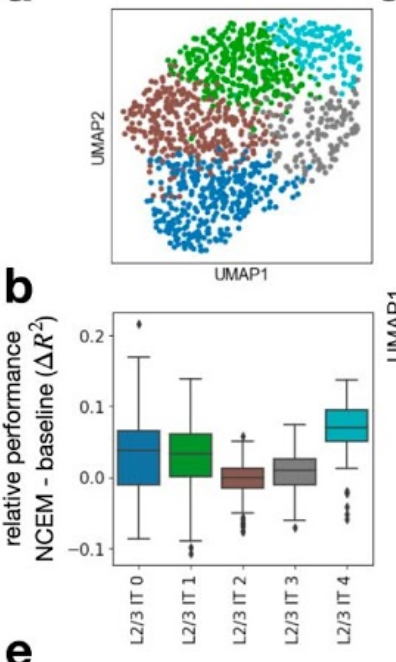

C

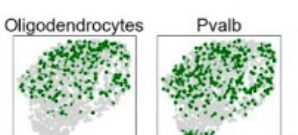

Vip
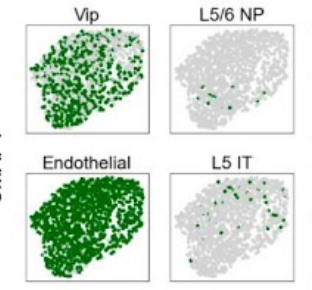

L2/3IT

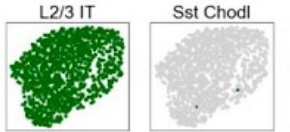

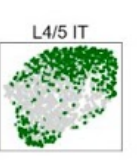

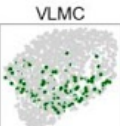

Sst

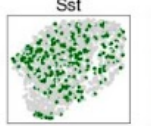

Pericytes

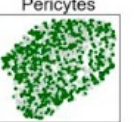

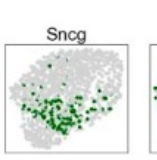

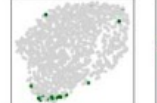

OPC
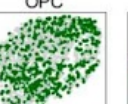

SMC

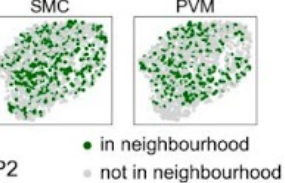

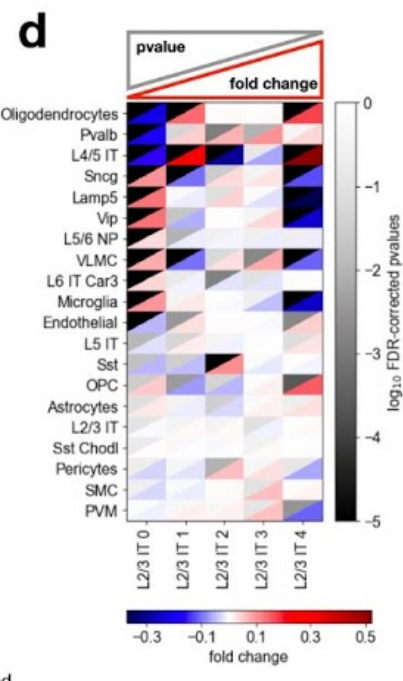
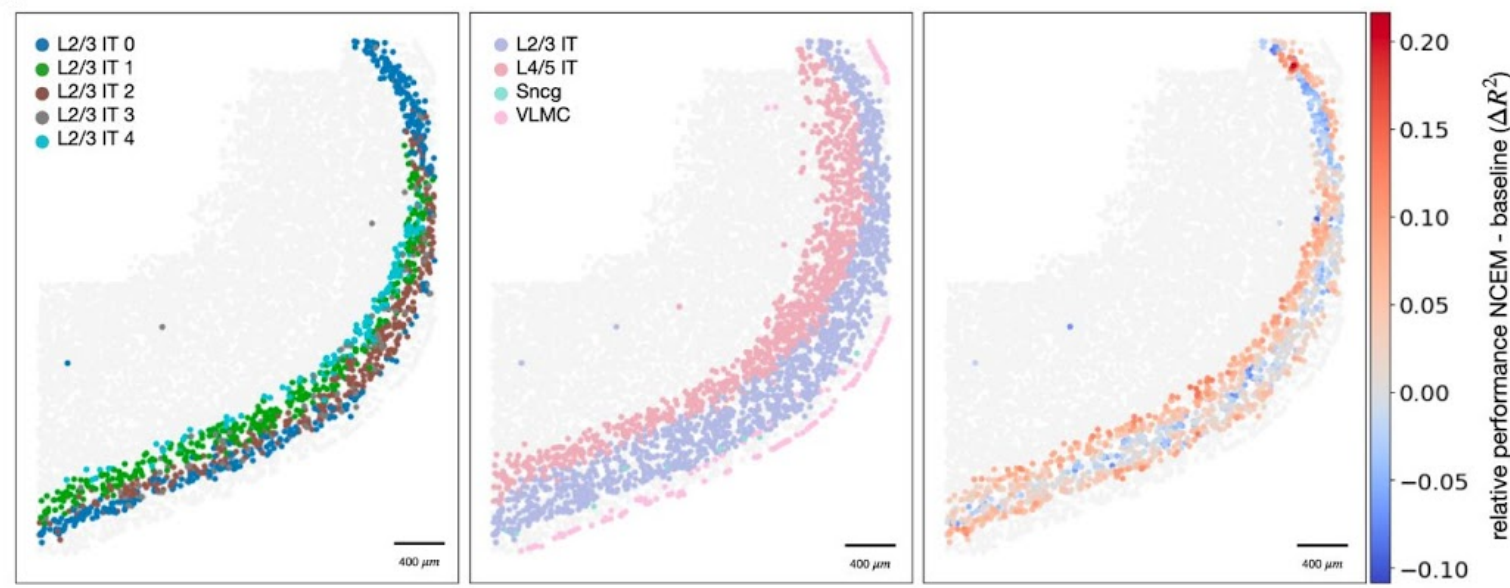

378 Figure 2: Cell heterogeneity can be attributed to niche composition. (a) UMAPs of molecular embedding of

379 L2/3 IT cells with molecular sub-clustering superimposed (colors as in b). (b) Distribution of cell-wise difference of

$380 R^{2}$ between NCEM and non-spatial baseline model by molecular sub-cluster (L2/3 IT 0: $n=316, L 2 / 3$ IT 1: $n=$

381314 , L2/3 IT 2: $n=313$, L2/3 IT 3: $n=133$, L2/3 IT 4: $n=128)$. The centerline of the boxplots defines the mean

382 over all relative $R^{2}$ values, the height of the box is given by the interquartile range (IQR), the whiskers are given

383 by 1.5 * IQR and outliers are given as points beyond the minimum or maximum whisker. (c) UMAPs of molecular

384 embedding of all L2/3 IT cells in an example image $(n=1204$ cells) showing if a given cell-type is present in the

385 neighborhood. The underlying neighborhoods were defined at the optimal resolution defined in Fig. $1 \mathrm{~d}(100 \mu \mathrm{m})$.

386 (d) Heatmap of fold change versus false-discovery rate corrected p-values of cluster enrichment of binary

387 neighborhood labels, where fold changes are the ratio between the relative neighboring source cell-type

388 frequencies per subtype cluster and the overall source cell-type frequency in the image. (e) Spatial allocation of

389 slice 153 of mouse brain in the MERFISH - brain dataset with L2/3 IT sub-states superimposed, L2/3 IT, L4/5 IT,

390 Sncg, and VLMC superimposed and superimposed the difference of $R^{2}$ between the NCEM at resolution of 100

$391 \mu \mathrm{m}$ and the best nonspatial baseline model. 
bioRxiv preprint doi: https://doi.org/10.1101/2021.07.11.451750; this version posted July 12, 2021. The copyright holder for this preprint (which was not certified by peer review) is the author/funder, who has granted bioRxiv a license to display the preprint in perpetuity. It is made available under aCC-BY 4.0 International license.

a

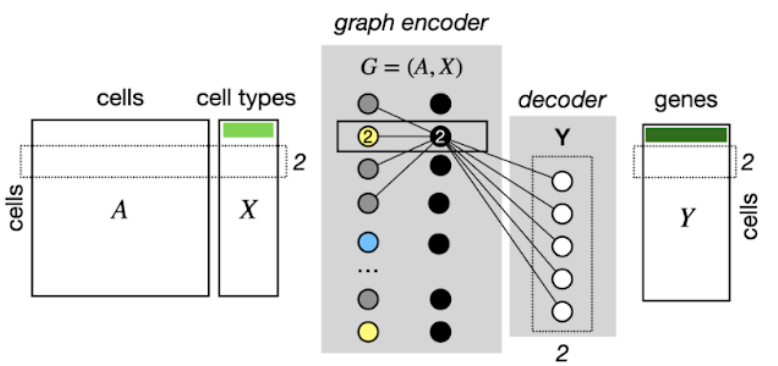

b

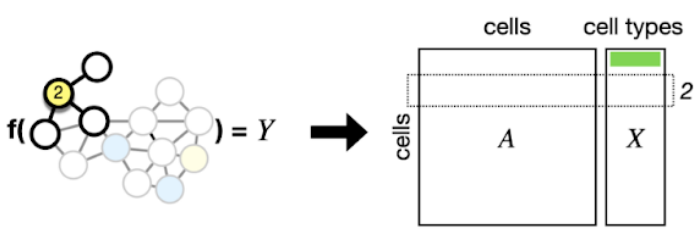

MERFISH - brain
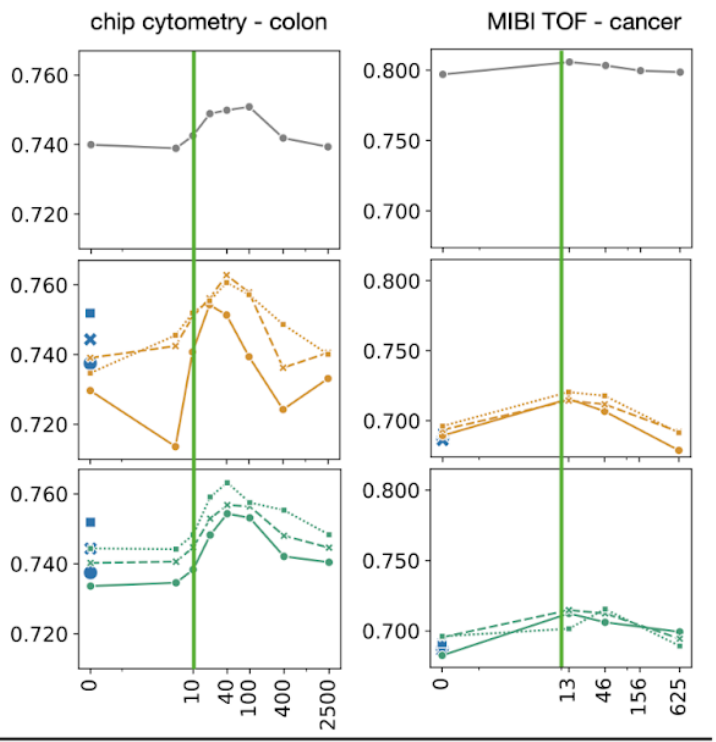

example

cell radius

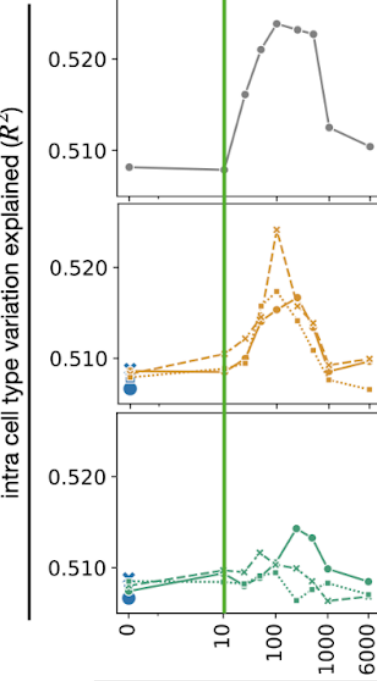

$\frac{1}{0}$

resolution in $\mu \mathrm{m}$

model:

$\rightarrow$ (interaction)

model:

- baseline

- ED-NCEM IND

split:

$\rightarrow 1$

$-*-2$
$-. * . .3$

model:

- baseline

— ED-NCEM GCN

split:

$\rightarrow 1$

$-0-2$

c

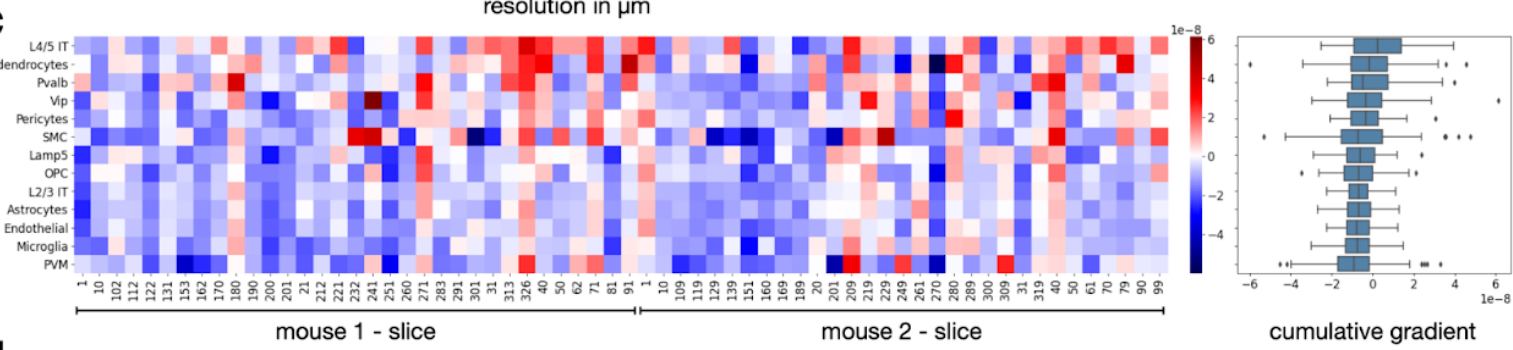

d
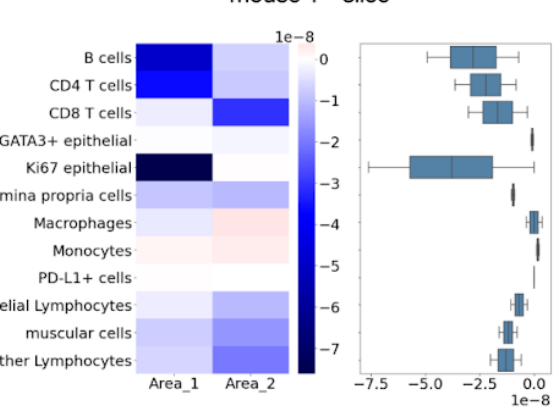

cumulative gradient

393 Figure 3: Nonlinear models of spatial dependencies of expression states. (a) A node-supervised model in

394 which the supervision label is the expression vector of a cell and the input consists of categorical cell-type

395 assignments and a spatial neighborhood graph. This model can also be viewed as an encoder-decoder model.

396 The encoder performs a graph-based embedding of a single cell and the decoder translates a bottleneck

397 activation vector into an expression state. (b) Nonlinear spatial dependencies in single-cell datasets. Shown are

398 the $\mathrm{R}^{2}$ values for held-out test data of encoder-decoder models by resolution in $\mu \mathrm{m}$ with cross validation

399 indicated as point shape and line style and comparatively mean performance of linear model in Fig. 1d. Linear

400 (interaction) (gray line): linear model with interaction effects; ED: encoder-decoder model; IND: the graph

401 convolution is an indicator function across cell-types in the neighborhood (yellow lines); GCN: the graph

402 convolution is a linear embedding (filter) of the cell-types in the neighborhood (teal lines); split (point shapes):

403 cross-validation split. (c) Heatmap of cumulative gradients (saliency) of gene expression prediction of L2/3 IT 
bioRxiv preprint doi: https://doi.org/10.1101/2021.07.11.451750; this version posted July 12, 2021. The copyright holder for this preprint (which was not certified by peer review) is the author/funder, who has granted bioRxiv a license to display the preprint in perpetuity. It is made available under aCC-BY 4.0 International license.

404 with respect to the input cells, aggregated by the sender cell-type clusters, on test data. Shown is a cumulative 405 gradient matrix of L2/3 IT predictions by source cell-type and image. (d) Heatmap of cumulative gradients 406 (saliency) of gene expression prediction of CD8 T cells with respect to the input cells, aggregated by the sender 407 cell-type cluster, on the test data. Shown is a cumulative gradient matrix of CD8 T cell predictions by source 408 cell-type and image. The cumulative absolute gradients in (c, d) are derived from the absolute gradients tensor 409 across each cell's molecular vector prediction with respect to the cells in the neighborhood (source cells) per 410 image (tensor shape: genes $\times$ cells $\times$ images), by taking a sum across the molecular output features and by

411 taking a sum across source cells of the same type (tensor shape: cell-types $\times$ images). For each box in (c, d),

412 the centerline defines the mean over all image-wise saliencies, the width of the box is given by the interquartile 413 range (IQR), the whiskers are given by $1.5 * I Q R$ and outliers are given as points beyond the minimum or 414 maximum whisker.

415
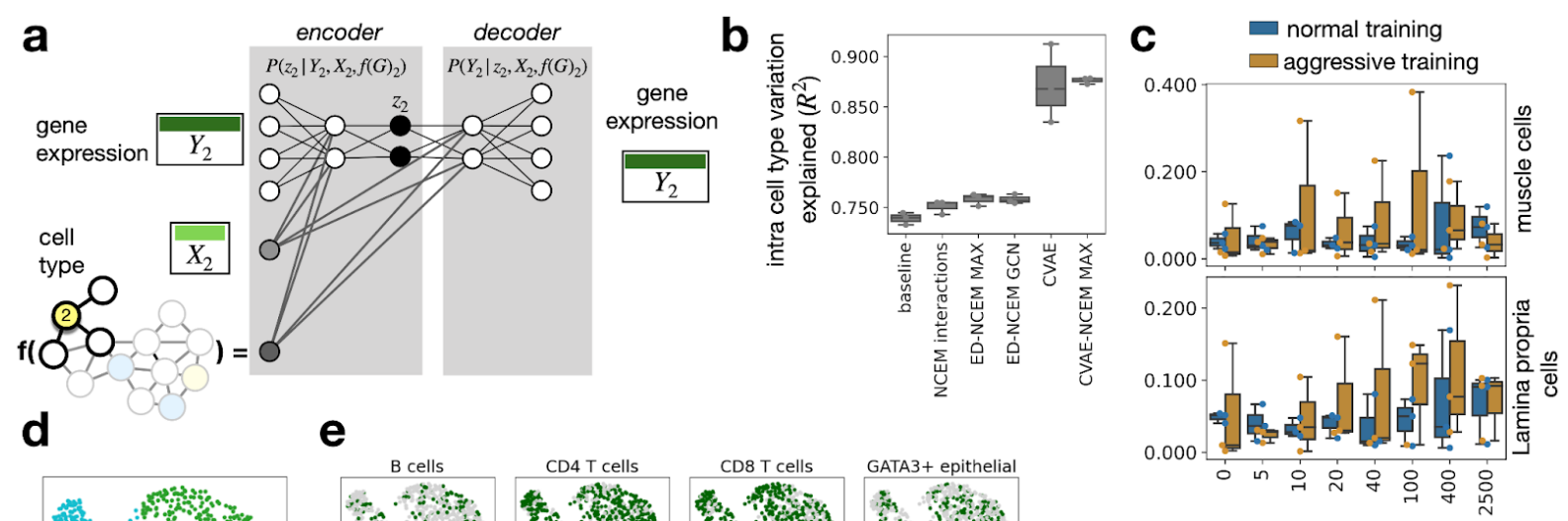

416

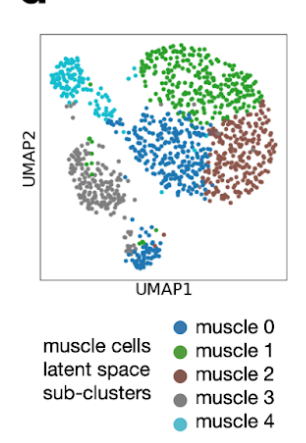

e
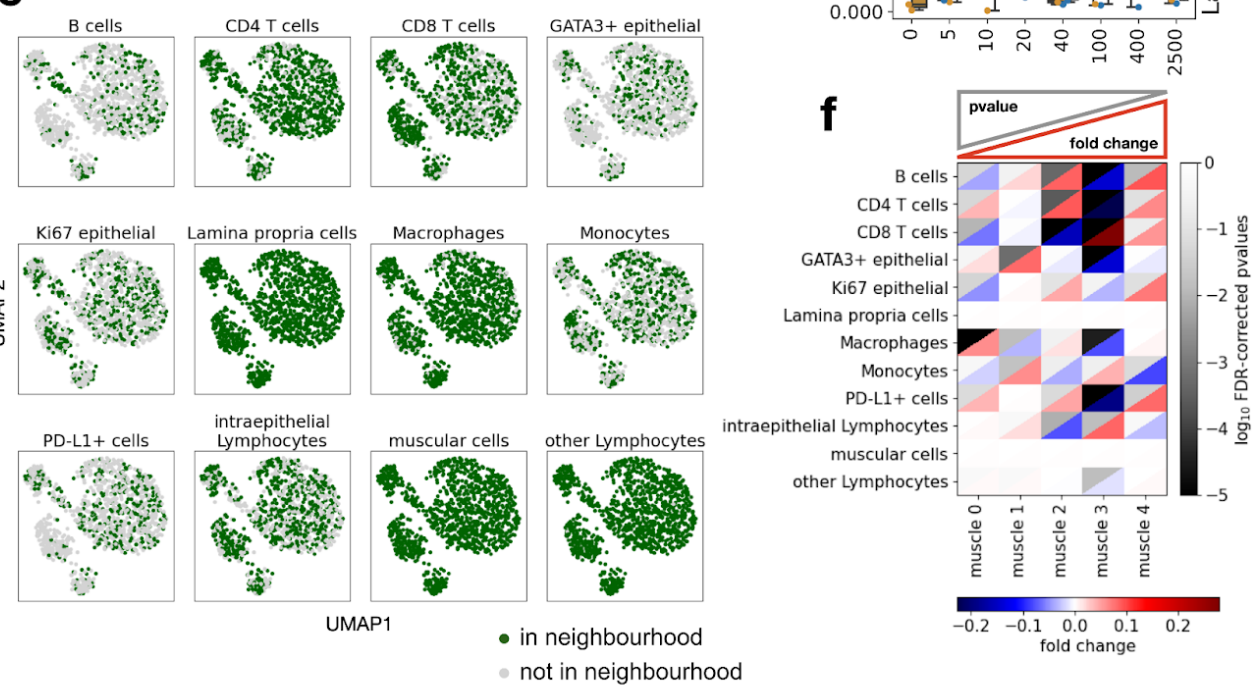

417 Figure 4: Modelling intrinsic and extrinsic variation in deep latent variable models. (a) A node generative

418 network (CVAE-NCEM) is a conditional variational autoencoder in which the condition is not a constant but a

419 graph embedding, which is also learned. (b) Latent variable models improve reconstructive performance. Shown

420 are the $R^{2}$ values of held-out test data based on the forward pass model evaluation from chip cytometry - colon

421 data for linear models, encoder-decoder models, and variational autoencoders for both NCEM and nonspatial

422 models. baseline: a nonspatial linear model of gene expression per cell-type; NCEM interactions: linear model

423 with interaction effects; $E D$ : encoder-decoder model; IND: the graph convolution is an indicator function across

424 cell-types in the neighborhood; GCN: the graph convolution is a linear embedding (filter) of the cell-types in the

425 neighborhood. (c) Neighborhood transfer performance of NCEM and nonspatial models. Shown is the $\mathrm{R}^{2}$ over

426 cells in the test set for models trained on predicting muscular cells and Lamina propria cells for both CVAE and

427 CVAE-NCEMs trained on neighborhoods with different radii with optimization algorithm as color. Plain: normal

428 CVAE training; aggressive: aggressive encoder training. For each box in (b, c), the centerline defines the mean

429 over all three cross-validations, the height of the box is given by the interquartile range (IQR), the whiskers are

430 given by 1.5 * IQR and outliers are given as points beyond the minimum or maximum whisker. (d-f) Latent

431 variables of CVAE-NCEM are confounded with neighborhood conditions. (d) UMAP of molecular embedding in

432 the CVAE-NCEM IND latent space of muscular cells in an example image $(n=1,149$ cells) with molecular

433 sub-clustering superimposed (muscle $0: n=315$, muscle $1: n=287$, muscle $2: n=238$, muscle $3: n=183$, 
bioRxiv preprint doi: https://doi.org/10.1101/2021.07.11.451750; this version posted July 12, 2021. The copyright holder for this preprint (which was not certified by peer review) is the author/funder, who has granted bioRxiv a license to display the preprint in perpetuity. It is made available under aCC-BY 4.0 International license.

434 muscle 4: $n=126$ ). (e) UMAPs of molecular embedding in the CVAE-NCEM IND latent space of all muscle cells

435 in the same image with superimposed binary label of presence of a given cell-type, as defined in the title, in the

436 neighborhood. The underlying neighborhoods were defined at a resolution of $100 \mu \mathrm{m}$. (f) Heatmap of fold change

437 versus false-discovery corrected p-values of cluster enrichment of binary neighborhood labels where fold

438 changes are the ratio between the relative neighboring source cell-type frequencies per subtype cluster and the

439 overall source cell-type frequency in the image. 
442 MERFISH - brain:

443 Zhang et al. ${ }^{12}$ measured mouse primary motor cortex with multiplexed error-robust fluorescence in situ 444 hybridization (MERFISH) in 634 images across two mice with 254 genes observed in 284,098 cells. The 445 cell-types originally annotated by Zhang et al., that are also used here, are astrocytes, endothelial, L2/3 446 intra-telencephalic (IT) neurons, L4/5 IT, L5/6 near-projecting (NP) neurons, L5 IT, L5 pyramidal tract (PT) 447 neurons, L6 cortico-thalamic (CT) projection neurons, L6 IT, L6 IT Car3, L6b, Lamp5, microglia, oligodendrocyte 448 precursor cells (OPC), oligodendrocytes, perivascular macrophages (PVM), pericytes, Parvalbumin (Pvalb), 449 smooth muscle cells (SMC), Sncg, somatostatin (Sst), Sst Chodl, vascular leptomeningeal cells (VLMC), 450 Vasoactive intestinal polypeptide (Vip), and other cells are annotated, where L identifies the layer (L1-L6) of the 451 distinctive laminar structure based on cytoarchitectural features (Supp. Fig. 1a). Pvalb, Sst, Vip, Sncg and Lamp5

452 define five subclasses of GABAergic cells. We removed cells labeled as "other" from the dataset. The gene-wise 453 mean-variance relationship does not indicate count noise (Supp. Fig. 13a). As domain information, we used an 454 identifier for the respective mouse. The dataset has a lateral resolution of $109 \mathrm{~nm}$ per pixel. Zhang et al. used a 455 seeded watershed algorithm to identify cell segmentation boundaries in each image. They performed 456 graph-based Louvain community detection ${ }^{19}$ with the first 35 principal components using Scanpy ${ }^{17}$ for $k=10$ 457 neighbors for cell-type clustering.

458 chip cytometry - colon:

459 Jarosch et al. measured an inflamed colon with chip cytometry in two images across one patient with 19 genes 460 observed in 11,321 cells. The cell-types originally annotated by Jarosch et al., which are modeled here, are B 461 cells, CD4 T cells, CD8 T cells, GATA3+ epithelial, Ki67 high epithelial, Ki67 low epithelial, lamina propria cells, 462 macrophages, monocytes, PD-L1+ cells, intraepithelial lymphocytes, muscular cells and other lymphocytes are 463 annotated (Supp. Fig. 1a). We coarsened the cell-type annotation by combining Ki67 high epithelial and Ki67 low 464 epithelial to a joined annotation of Ki67 epithelial. The gene-wise mean-variance relationship indicates count 465 noise (Supp. Fig. 13b). Therefore, we log-transformed gene expression values. Jarosch et al. performed 466 thresholding, watershed algorithm and additionally implemented a cell-type-specific segmentation method to 467 segment individual cells. Intensity values were corrected for spatial spillover prior to quantification. Cell-types

468 were clustered using the Leiden clustering ${ }^{18}$ of the neighborhood-graph.

469 MIBI TOF - cancer:

470 Hartmann et al. ${ }^{8}$ measured colorectal carcinoma and healthy adjacent tissue with multiplexed ion beam imaging 471 by time-of-flight (MIBI-TOF) in 58 images across four individuals with 36 genes observed in 63,747 cells. The 472 cell-types originally annotated by Hartmann et al., which are modeled here, are endothelial, epithelial, fibroblast, 473 CD11c myeloid, CD68 myeloid, CD4 T cells, CD8 T cells and other immune cells are annotated (Supp. Fig. 1a). 474 A coarser cell-type labeling was not applied to this dataset. The cohort in this dataset includes two patients with 475 colorectal carcinoma and two healthy controls. The images have a size of $400 \mu \mathrm{m}^{2}$ and $1,024 \times 1,024$ pixels. The 476 gene-wise mean-variance relationship does not indicate count noise (Supp. Fig. 13b). We scaled the model 477 outputs by cell-wise size factors. Hartmann et al. trained a convolutional neural network. They fed the output into 478 the watershed algorithm to segment individual cells and cell-types were clustered using the FlowSOM R package 479 and manually annotated based on their lineage marker profiles.

480 MELC - tonsils:

481 Pascual-Reguant et al. $^{7}$ measured tonsils from patients undergoing tonsillectomy with multi-epitope ligand 482 cartography (MELC), an immunohistochemistry approach, in one image across one patient, with 51 genes 483 observed in 9,512 cells. The cell-types originally annotated by Pascual-Reguan et al., which are modeled here, 484 are B cells, endothelial cells, ILC, monocytes/macrophages/DC, NK cells, plasma cells, T cytotoxic cells, T helper 485 cells and other cells are annotated (Supp. Fig. 1a). We removed cells labeled as "other" from the dataset. The 486 gene-wise mean-variance relationship does not indicate count noise (Supp. Fig. 13d). Pascual-Reguant et al. 487 performed segmentation by applying a signal-classification step using llastik 1.3.2 and an object-recognition step 488 using CellProfiler 3.1.8, which were analyzed and clustered in Orange 3.26.0. 


\section{CODEX - cancer:}

490 Schürch et al. ${ }^{13}$ measured advanced-stage colorectal cancer with co-detection by indexing (CODEX) in 140 491 images across 35 patients with 57 genes observed in 272,266 cells. The cell-types originally annotated by Zhang 492 et al., which are modeled here, are B cells, CD11b+ monocytes, CD11c+ dendritic cells, CD11b+CD68+ 493 macrophages, CD163+ macrophages, CD68+ macrophages, CD68+ macrophages GzmB+, CD68+CD163+ 494 macrophages, CD3+ $T$ cells, CD4+ $T$ cells, CD4+ T cells CD45RO+, CD4+ T cells GATA3+, CD8+ T cells, NK 495 cells, T regs, adipocytes, dirt, granulocytes, immune cells, immune cells / vasculature, lymphatics, nerves, 496 plasma cells, smooth muscle, stroma, tumor cells, tumor cells / immune cells and vasculature are annotated 497 (Supp. Fig. 1a). Cells with an annotation of dirt or an undefined label were removed from the dataset. A coarser 498 cell-type grouping was applied to the macrophage groups CD11b+CD68+ macrophages, CD163+ macrophages, 499 CD68+ macrophages, CD68+ macrophages GzmB+ and CD68+CD163+ macrophages. Additionally, CD4+ T

500 cells, CD4+ $T$ cells $C D 45 R O+$ and $C D 4+T$ cells GATA3+ were grouped into CD4+ $T$ cells. The gene-wise 501 mean-variance relationship indicates count noise (Supp. Fig. 13e). Therefore, we scaled model outputs by the 502 node size in the respective output layer of each model class. Schürch et al. performed segmentation using the 503 CODEX toolkit segmenter and unsupervised cell-type clustering using X-shift.

504

\begin{tabular}{|c|cccccc}
$\begin{array}{c}\text { dataset } / \\
\text { first author }\end{array}$ & technology & domain & transform & $\begin{array}{c}\text { node size } \\
\text { scaling }\end{array}$ & batch size \\
\hline Zhang & MERFISH & patient & - & False & 64 \\
\hline Jarosch & Chip Cytometry & patient & $\log (x+1)$ & False & 2 & 100 \\
\hline Hartmann & MIBI-TOF & image & - & True & 58 & 10 \\
\hline Pascual-Reguant & MELC & patient & - & True & 1 & 200 \\
\hline Schürch & CODEX & patient & $\log (x+1)$ & True & 140 & 10
\end{tabular}

506 Table 1: Overview of datasets analyzed in this study. Shown are the spatial molecular profiling chemistry, the 507 domain effect accounted for via batch covariates, the data transform used on the expression vectors, the 508 inclusion of cell size factors, the number of images given to the models during each update (batch size) and the 509 number of nodes evaluated per image per batch (n).

\section{Models}

511 The inputs to NCEMs are a gene expression matrix $Y \in R^{N \times J}$, where $N$ is the number of cells and $J$ is the 512 number of genes with $y_{i}$ being the gene expression vector for gene $i=1, \ldots, J$, a matrix of observed cell-types $513 X_{l} \in R^{N \times L}$ where $L$ is the number of distinct cell-type labels and $X_{c} \in R^{N \times C}$ is a matrix specifying the batch 514 assignments, with $C$ being the number of distinct batches or domains, such as images or patients, in the 515 dataset. We denote the adjacency matrix of connected cells as $A \in R^{N \times N}$ which is calculated based on the spatial 516 proximity of cells per image. For linear models and models with an indicator aggregator, a binary adjacency 517 matrix is used with $a_{i j}=1$ if $d\left(x_{i}, x_{j}\right) \leq \delta_{\max }$ where $d(\cdot, \cdot)$ describes the euclidean distance between nodes $i, j \in N$ 518 and $\delta_{\max }$ is the maximal distance between interacting nodes (neighborhood size), and $a_{i j}=0$ otherwise. For 519 models using a GCN as graph layer, we normalize $A$ such that all rows sum to one, so $D^{-1} A$ where $D$ is the 520 diagonal node degree matrix. The output of NCEMs is $\hat{Y} \in R^{N \times J}$, a reconstruction of the input count matrix $Y$, 521 with $\hat{y}_{i}$ being the reconstructed expression for gene $i=1, \ldots, J$. For MIBI-TOF - cancer, MELC - tonsils and 522 CODEX - cancer, we applied size factor scaling to the network output $\hat{Y}$. Let $\bar{\mu}=\frac{1}{N} \sum_{i} \sum_{j}^{J} y_{i j}$ be the global mean per 523 node, then size factors are given by $s f_{k}=\bar{\mu} / \sum_{j}^{J} y_{j, k} \in R^{N}$. The network output scaling is then given by $\hat{Y}=\hat{Y} \cdot s f$.

525 Loss functions: According to the noise structure of the datasets explored in this paper (Supp. Fig. 13) we use a 526 Gaussian log-likelihood loss as an optimization objective for GLM and ED models with $527 \quad l_{N}=\frac{1}{N} \sum_{i=1}^{N}\left(\log \left(\sqrt{2 \pi} \cdot \sigma_{i}\right)+\frac{\left(\hat{(⿻)}_{i}-y_{i}\right)}{\sigma_{i}^{2}}\right)$, where $\sigma_{i}$ is the predicted standard deviation for a gene $i=1, \ldots, N$. The loss 528 function of CVAE models is the negative log-likelihood regularized by the Kullback-Leibler divergence between 

made available under aCC-BY 4.0 International license.

529 the encoder distribution $q_{\varphi}(z \mid Y)$ and the distribution of the latent space $p(z)$, so $530 l_{C V A E}=-l l_{N}+K L\left[q_{\varphi}(z \mid Y) \| p(z)\right]$. For chip cytometry - colon and CODEX - cancer we therefore $\log (Y+1)$ 531 transformed the gene expression data.

532

533 Optimization: For each dataset, we ran grid searches to find the optimal set of hyperparameters. As batch size

534 we choose the number of images per dataset. The number of nodes evaluated per image per batch was selected

535 to ensure convergence and stabilize training. All models were trained with the Adam optimizer algorithm

536 implemented in tensorflow. Linear models were trained with a $l r=0.5$, the remaining models were trained for a

537 varying learning rate of $l r=\{0.5,0.05,0.005\}$. Additionally, we used a learning scheduler on the validation loss

538 with a patience of 20 epochs which reduces the learning rate by a factor of 0.5 , so $l r_{n e w}=l r * 0.5$ and early

539 stopping with a patience of 100 epochs. The exact description of all grid searches in code are supplied in the

540 benchmarking repository (Code Availability).

541

542 Linear NCEM: The NCEM includes two linear regression models. The nonspatial baseline linear model infers a

543 reconstruction $\hat{Y}$ from a nodes cell-type and respective domain information via $\hat{Y}=X_{D} \beta$, where $X_{D}$ is the design

544 matrix and $\beta \in R^{(L+C) \times N}$ are the parameters learned by the model. The design matrix for nonspatial baseline

545 models is given by $X_{D}=\left(X_{l}, X_{c}\right) \in R^{N \times(L+C)}$. The spatial counterpart model, the NCEM, has access to an

546 interaction matrix. First, we compute discrete target cell interactions with $X_{T}=1_{\left(A \cdot X_{l}>0\right)} \in[0,1]^{N \times L}$, where $1_{(\cdot)}$

547 represents an indicator function. To generate a matrix representation of target-source cell interactions, we

548 compute the interaction between each column of $X_{l}$ and each column $X_{T}$ via the point-wise product.The

549 resulting interaction matrix is then denoted as $X_{T S} \in\{0,1\}^{N \times L^{2}}$ and the design matrix for the linear model with

550 interaction terms is given by $X_{D}=\left(X_{l}, X_{T S}, X_{c}\right) \in R^{N \times\left(L+L^{2}+C\right)}$. Equivalently, the model infers $\hat{Y}=X_{D} \beta$ where

$551 \beta \in R^{\left(L+L^{2}+C\right) \times N}$ and additionally the gene variance per node which is inferred in the last layer of the linear model.

552 We also considered an NCEM without interaction terms which does not have receiver-specific sender effects, but

553 only global sender effects which account for the presence of senders in the niche via $X_{S} \in\{0,1\}^{N \times L}$ :

$554 X_{D}=\left(X_{l}, X_{S}, X_{c}\right) \in R^{N \times(L+L+C)}$. Coefficient significance is computed with Wald hypothesis testing with a

555 significance threshold of $\tau=0.01$ on the parameters learned by the model for the interaction matrix $X_{T S}$.

556

557 Nonlinear encoder-decoder NCEM (ED-NCEM): The NCEM includes nonlinear encoder-decoder models that

558 encode the neighborhood through a graph neural network (ED-NCEM) and decode expression vectors. The

559 nonspatial null model is a nonlinear model (ED) that predicts expression from cell-type and graph-level

560 predictors, alone. An encoder is given by $f_{e n c}: q_{\varphi}\left(z \mid X_{l}, g\left(A, X_{l}\right), X_{c}\right)$, which encodes the cell-type labels $X_{l}$,

561 some graph-level predictors $X_{c}$ and the local graph embedding $g\left(A, X_{l}\right)$, based on the adjacency matrix $A$, into a

562 latent state $z$. The latent state is input to the decoder and given by $f_{d e c}: p_{\theta}\left(\hat{Y} \mid z, X_{l}, g\left(A, X_{l}\right), X_{c}\right)$. If $g\left(A, X_{l}\right)$ is

563 and indicator embedding function as described in the section "Linear NCEM", then $g\left(A, X_{l}\right)=X_{T S}$ and the input to

564 the linear model and the encoder are the same. If then also all hidden layers are removed from the ED-NCEM, a

565 single linear transformation of the input remains which is equivalent to the linear NCEM. Alternatively, $g\left(A, X_{l}\right)$

566 can be a graph embedding learned by a graph-convolutional network $(\mathrm{GCN})^{10,11}$. A one-layer GCN is given by

$567 g\left(A, X_{l}\right)=\operatorname{softmax}\left(\operatorname{Re} L U\left(\bar{A} X_{l} W\right)\right)$ where $W \in R^{L \times H}$ is an input-to-graph-embedding weight matrix with $H$ being

568 the dimension of the learned graph representation and $\bar{A}$ being the normalized adjacency matrix. In this case, $W$

569 can be learned using gradient descent.

570

571 Conditional variational autoencoder NCEM (CVAE-NCEM): A variational autoencoder ${ }^{29}$ learns a distribution over

572 node states $Y$ through a variational posterior over a latent space representation $z$ which yields a reconstruction

$573 \hat{Y}$ of $Y$ via a likelihood function (the decoder). The nonspatial CVAE null model contains the cell-type and

574 graph-level predictors as a condition in the variational posterior and the likelihood. In CVAE-NCEM, the

575 conditions are the cell-type labels $X_{l}$, some graph-level predictors $X_{c}$ and the local graph embedding $g\left(A, X_{l}\right)$, 
576 based on the adjacency matrix $A$. The encoder is then given by $f_{e n c}^{N G-C V A E}: q_{\varphi}\left(z \mid Y, X_{l}, g\left(A, X_{l}\right), X_{c}\right)$ and the 577 decoder is defined by $f_{d e c}^{N G-C V A E}: p_{\theta}\left(\hat{Y} \mid z, X_{l}, g\left(A, X_{l}\right), X_{c}\right)$. A CVAE-NCEM for a full dataset depends on both

578 the spatial context and the type of the cell itself. This setting presents the challenge of encountering a

579 non-identifiability between variance attributed to latent variables, cell-type conditions, and neighborhood context.

580 In this study, we consider the CVAE-NCEM trained on the molecular vectors of a single target cell-type as a

581 function of the full neighborhood context to remove the non-identifiability with respect to cell-type variation and

582 focus on the non-identifiability between latent variables and neighborhoods.

\section{Normalized saliency maps}

584 Saliency maps are used to differentiate the importance of features in the network input to analyze their

585 importance for the network output for nonlinear models. In our case, saliency maps are aggregated at the

586 cell-type level to extract communication events learned by the model, so $S A L S \in R^{L x L}$ with $L$ being the number

587 of distinct cell-types in the model. Non-normalized saliencies will show a pattern similar to the contact frequency

588 matrix $M \in R^{L x L}$ as cell-types with frequent connections will skew the learned importance of cell connections.

589 We therefore normalize the saliencies by the absolute frequency of cell-cell connections, that is

$590 S A L S_{\text {norm }}=\frac{1}{x_{i j}} * S A L S_{i j} \in R^{L x L}$ for $x_{i j} \in M$ with $i, j=1, \ldots, L$.

\section{Model evaluation}

592 Overall model performance evaluation is based on the coefficient of determination $R^{2}=1-\frac{\sum_{i=1}^{N}\left(y_{i}-\widehat{y}_{i}\right)^{2}}{\sum_{i=1}^{N}\left(y_{i}-\bar{y}\right)^{2}} \in[0,1]$ with

$593 \bar{y}$ being the mean over gene expression values. Best performing models are selected based on highest $R^{2}$ for

594 the validation dataset. These metrics are based on model predictions, which derive from a forward pass through

595 the model. The performance of CVAEs is additionally assessed in style transfer tasks. In style transfer, the gene

596 expression state and neighborhood of a reference node from the source domain is encoded to estimate the latent

597 states of this node. This latent representation is then decoded to the target domain, which implies conditioning

598 the decoding on the target neighborhood:

$599 z^{i} \sim q_{\varphi}\left(z \mid Y^{i}, X_{l}^{i}, g\left(A, X_{l}\right), X_{c}^{i}\right)(1)$

$600 \hat{Y^{j}}=p_{\theta}\left(z^{i}, X_{l}^{j}, g\left(A, X_{l}\right), X_{c}^{j}\right)(2)$

601 where $i, j$ are cell indices, $q_{\varphi}$ is the variational posterior and $p_{\theta}$ is the decoder network. See also "Conditional

602 variational autoencoder NCEM (CVAE-NCEM)" for details on the notation.

\section{Ligand-receptor association analysis}

604 For ligand-receptor permutation tests we used the CellphoneDB ${ }^{3}$ implementation in Squidpy ${ }^{15}$. For the chip 605 cytometry - colon, MIBI TOF - cancer $^{8}$, MELC - tonsils ${ }^{7}$, and CODEX - cancer ${ }^{13}$ datasets, node feature names 606 are mapped to HGNC gene names. After the mapping, we used the ligand-receptor interaction pairs of the 607 Omnipath database ${ }^{30}$ in Squidpy ${ }^{21}$ and ran the permutation test for $n=1,000$ permutations. For three datasets, 608 we used a random subsample of all cells (MERFISH - brain ${ }^{12} 10 \%$ with $n=27,655$, MIBI TOF - cancer $^{8} 40 \%$ 609 with $n=25,498$ and CODEX - cancer $^{13} 10 \%$ with $\left.n=25,186\right)$. Results are visualized with Squidpy ${ }^{21}$ with only

610 p-values below a threshold of 0.3 shown.

\section{Variance attribution analysis}

612 We used Uniform Manifold Approximation and Projection ${ }^{31}$ (UMAP) to embed the cells in two dimensions for 613 visualization of high-dimensional data. For the UMAPs of the MERFISH - brain data ${ }^{12}$ matrix (Fig. 1c and Supp. 614 Fig. 5b) we performed dimensionality reduction using PCA with the first 35 principal components (PCs) and the 615 nearest neighborhood size of $k=10$. A similar approach was described by Zhang et al. to identify stable clusters 616 for subsequent cell-type annotation. For the UMAPs of L2/3 IT neurons in slice 153 (Fig. 2) and slice 162 (Supp. 617 Fig 5b) of the MERFISH - brain dataset, we used the first 40 PCs with $k=40$ and performed graph-based 618 Louvain community detection ${ }^{19}$ using Scanpy ${ }^{17}$ to define stable L2/3 IT substates. For the UMAPS of CD8 T cells 619 in area 1 in the chip cytometry dataset (Supp. Fig. 8), we used the input matrix directly and $k=22$. For the 
620 UMAPs of CD8 T cells in image 1, 5, 8 and 16 of the MIBI-TOF - cancer dataset (Supp. Fig. 9), we used the input

621 matrix directly and $\mathrm{k}=60$. Clustering of the latent space in CVAE and CVAE-NCEM IND models (Fig. 4d, Supp.

622 Fig. 11c) was performed using the latent space matrix directly and k = 80 for the MERFISH - brain dataset and $k$

$623=250$ for the chip cytometry - colon dataset.

624

625 For cluster enrichment analysis, we performed Fisher's exact test. Each contingency table is composed of two

626 categorical variables. The first variable is the number of cells in one specific L2/3 IT substate versus the

627 remaining L2/3 IT substates. The second variable is the number of cells with a respective source type in their

628 neighborhood and those cells where this source type is not present in the neighborhood. We performed

629 Benjamini and Hochberg false discovery rate correction (FDR) of cluster enrichment p-values. A similar approach

630 was used for the cluster enrichment analysis of CD8 T cells in the chip cytometry - colon and the MIBI-TOF -

631 cancer datasets.

632

633 For comparison of the source cell-type rankings of contact frequencies, ligand-receptor analysis and cluster

634 enrichment analysis of L2/3 IT cells, we performed Kendall's tau correlation analysis and computed the tau

635 statistic and the two-sided p-value. Contact frequencies are ranked by source type frequencies (row L2/3 IT of

636 Supp. Fig. 6). For ligand-receptor analyses, source cell-types are ranked based on the number of significant

637 p-values below a threshold of 0.05 for this L2/3 IT interaction. For the cluster enrichment analysis we used the

638 ranking shown in Fig. 2d.

639 Variance decomposition into inter- and intra-cell-type variance

640 The variance of a single-cell resolved dataset can be decomposed into inter-cell-type variance, intra-cell-type

641 variance, and gene variance. The gene variance is independent of cell-type definitions and can therefore be

642 considered separately from relative intra- and inter-cell-type variance.

$$
\begin{aligned}
& \operatorname{var}(x)=\sum_{i}\left(x_{i}-\bar{x}\right)^{2}=\sum_{i}\left(x_{i}-\bar{x}_{k(i)}^{g}+\bar{x}_{k(i)}^{g}-\bar{x}^{g}\right)^{2} \\
= & \sum_{i}\left(x_{i}-\bar{x}_{k(i)}^{g}\right)^{2}+\sum_{i}\left(\bar{x}_{k(i)}^{g}-\bar{x}^{g}\right)^{2}-\sum_{k} 2 *\left(\bar{x}_{k}^{g}-\bar{x}^{g}\right) \sum_{i \forall k(i)=k}\left(x_{i}-\bar{x}_{k}^{g}\right) \\
643= & \sum_{i}\left(x_{i}-\bar{x}_{k(i)}^{g}\right)^{2}+\sum_{i}\left(\bar{x}_{k(i) g}+\bar{x}^{g}-\bar{x}^{g}-\bar{x}\right)^{2} \\
= & \sum_{\text {intra cell type variance }}^{\sum_{i}\left(x_{i}-\bar{x}_{k(i)}\right)^{2}}+\underbrace{\sum_{i}\left(\bar{x}_{k(i)^{g}}-\bar{x}^{g}\right)^{2}}_{\text {inter cell type variance }}+\underbrace{\sum_{i}\left(\bar{x}^{g}-\bar{x}\right)^{2}}_{\text {gene variance }}
\end{aligned}
$$

644 where $x_{\mathrm{i}}$ is the expression vector of cell $i, \bar{x}^{g}$ is the vector of gene-wise means of the dataset, $\bar{x}_{k}^{g}$ is the vector of

645 gene-wise means of the cells in cell-type $k, k(i)$ is the cell-type of cell $i$, and $\bar{x}$ is the scalar gene- and cell-wise

646 mean of the dataset.

\section{Data Availability}

648 The MERFISH - brain $^{12}$, MIBI TOF - cancer ${ }^{8}$, MELC - tonsils ${ }^{7}$ and CODEX - cancer ${ }^{13}$ datasets are publicly

649 available (Online Methods). The chip cytometry - colon dataset has been generated by the Busch lab and is

650 currently under review, and has kindly been provided to us.

\section{Code Availability}

652 All models described here are implemented in a Python package available at https://github.com/theislab/ncem.

653 All benchmarking and analysis codes are provided at https://github.com/theislab/ncem benchmarks. Tutorials for

654 model usage are available from https://github.com/theislab/ncem tutorials.

\section{Author Contributions}

656 DSF and FJT conceived the project. DSF and ACS performed the analysis and wrote the code. DSF, ACS and

657 FJT wrote the manuscript. 


\section{Acknowledgements}

659 We would like to thank Sabrina Richter, Mohammad Lotfollahi, Prof. Dr. Stephan Günnemann, Dr. Christian M.

660 Schürch, Sebastian Jarosch and Prof. Dr. Dirk Busch for valuable discussion and feedback on this project. In

661 particular, we want to thank Sebastian Jarosch and Prof. Dr. Dirk Busch for sharing the chip cytometry - colon

662 dataset pre-publication. We would like to thank Dr. Luke Zappia and Giovanni Palla for their valuable feedback on

663 this manuscript.

664

665 This work was supported by the German Federal Ministry of Education and Research (BMBF) under Grant No.

666 01IS18036B and No. 01IS18053A, by the Bavarian Ministry of Science and the Arts in the framework of the

667 Bavarian Research Association "ForInter" (Interaction of human brain cells), by the Wellcome Trust Grant

668 108413/A/15/D and by the Helmholtz Association's Initiative and Networking Fund through Helmholtz Al [grant

669 number: ZT-I-PF-5-01]. D.S.F. acknowledges support from a German Research Foundation (DFG) fellowship

670 through the Graduate School of Quantitative Biosciences Munich (QBM) [GSC 1006 to D.S.F.] and by the

671 Joachim Herz Foundation. ACS has been funded by the German Federal Ministry of Education and Research

672 (BMBF) under Grant No. 01IS18036B.

\section{Conflict of interest}

674 F.J.T. reports receiving consulting fees from Cellarity Inc., and ownership interest in Cellarity, Inc. and

675 Dermagnostix.

\section{References}

677 1. Raposo, G. \& Stoorvogel, W. Extracellular vesicles: exosomes, microvesicles, and friends. J. Cell Biol. 200,

678 373-383 (2013).

679 2. Browaeys, R., Saelens, W. \& Saeys, Y. NicheNet: modeling intercellular communication by linking ligands to

680 target genes. Nat. Methods 17, 159-162 (2020).

681 3. Efremova, M., Vento-Tormo, M., Teichmann, S. A. \& Vento-Tormo, R. CellPhoneDB: inferring cell-cell

682 communication from combined expression of multi-subunit ligand-receptor complexes. Nat. Protoc. 15,

683 1484-1506 (2020).

684 4. Almet, A. A., Cang, Z., Jin, S. \& Nie, Q. The landscape of cell-cell communication through single-cell

685 transcriptomics. Current Opinion in Systems Biology vol. 26 12-23 (2021).

686 5. Arnol, D., Schapiro, D., Bodenmiller, B., Saez-Rodriguez, J. \& Stegle, O. Modeling Cell-Cell Interactions from

687 Spatial Molecular Data with Spatial Variance Component Analysis. Cell Rep. 29, 202-211.e6 (2019).

688 6. Goltsev, Y. et al. Deep Profiling of Mouse Splenic Architecture with CODEX Multiplexed Imaging. Cell 174,

689 968-981.e15 (2018).

690 7. Pascual-Reguant, A. et al. Multiplexed histology analyses for the phenotypic and spatial characterization of

691 human innate lymphoid cells. Nat. Commun. 12, 1737 (2021).

692 8. Hartmann, F. J. et al. Single-cell metabolic profiling of human cytotoxic T cells. Nat. Biotechnol. 39, 186-197

693 (2021).

694 9. Xia, C., Fan, J., Emanuel, G., Hao, J. \& Zhuang, X. Spatial transcriptome profiling by MERFISH reveals

695 subcellular RNA compartmentalization and cell cycle-dependent gene expression. Proc. Natl. Acad. Sci. U.

696 S. A. 116, 19490-19499 (2019). 
10. Kipf, T. N. \& Welling, M. Semi-Supervised Classification with Graph Convolutional Networks. arXiv [cs.LG]

698

699

700

701

702

703 (2016).

11. Scarselli, F., Gori, M., Tsoi, A. C., Hagenbuchner, M. \& Monfardini, G. The graph neural network model. IEEE Trans. Neural Netw. 20, 61-80 (2009).

12. Zhang, M. et al. Molecular, spatial and projection diversity of neurons in primary motor cortex revealed by in situ single-cell transcriptomics. doi:10.1101/2020.06.04.105700.

13. Schürch, C. M. et al. Coordinated Cellular Neighborhoods Orchestrate Antitumoral Immunity at the Colorectal Cancer Invasive Front. Cell 183, 838 (2020).

14. Kleshchevnikov, V. et al. Comprehensive mapping of tissue cell architecture via integrated single cell and spatial transcriptomics. doi:10.1101/2020.11.15.378125.

15. Palla, G., Spitzer, H., Klein, M., Fischer, D. \& Schaar, A. C. Squidpy: a scalable framework for spatial single cell analysis. bioRxiv (2021).

16. Hetzel, L., Fischer, D. S., Günnemann, S. \& Theis, F. J. Graph Representation Learning for Single Cell Biology. Current Opinion in Systems Biology (2021) doi:10.1016/j.coisb.2021.05.008.

17. Wolf, F. A., Angerer, P. \& Theis, F. J. SCANPY: large-scale single-cell gene expression data analysis. Genome Biol. 19, 15 (2018).

18. Traag, V. A., Waltman, L. \& van Eck, N. J. From Louvain to Leiden: guaranteeing well-connected communities. Sci. Rep. 9, 5233 (2019).

19. Blondel, V. D., Guillaume, J.-L., Lambiotte, R. \& Lefebvre, E. Fast unfolding of communities in large networks. J. Stat. Mech. 2008, P10008 (2008).

20. Ishida, Y., Agata, Y., Shibahara, K. \& Honjo, T. Induced expression of PD-1, a novel member of the immunoglobulin gene superfamily, upon programmed cell death. The EMBO Journal vol. 11 3887-3895 (1992).

21. Lopez, R., Regier, J., Cole, M. B., Jordan, M. I. \& Yosef, N. Deep generative modeling for single-cell transcriptomics. Nat. Methods 15, 1053-1058 (2018).

22. Eraslan, G., Simon, L. M., Mircea, M., Mueller, N. S. \& Theis, F. J. Single-cell RNA-seq denoising using a deep count autoencoder. Nat. Commun. 10, 390 (2019).

23. Lotfollahi, M., Wolf, F. A. \& Theis, F. J. scGen predicts single-cell perturbation responses. Nat. Methods 16, 715-721 (2019).

24. Alemi, A. A. et al. Fixing a Broken ELBO. arXiv [cs.LG] (2017).

25. He, J., Spokoyny, D., Neubig, G. \& Berg-Kirkpatrick, T. Lagging Inference Networks and Posterior Collapse in Variational Autoencoders. arXiv [cs.LG] (2019).

26. Giesen, C. et al. Highly multiplexed imaging of tumor tissues with subcellular resolution by mass cytometry. Nat. Methods 11, 417-422 (2014). 
bioRxiv preprint doi: https://doi.org/10.1101/2021.07.11.451750; this version posted July 12, 2021. The copyright holder for this preprint

(which was not certified by peer review) is the author/funder, who has granted bioRxiv a license to display the preprint in perpetuity. It is made available under aCC-BY 4.0 International license.

731 27. Eng, C.-H. L. et al. Transcriptome-scale super-resolved imaging in tissues by RNA seqFISH. Nature vol. 568 $732 \quad$ 235-239 (2019).

733 28. Veličković, P. et al. Graph Attention Networks. arXiv [stat.ML] (2017).

734 29. Kingma, D. P. \& Welling, M. Auto-Encoding Variational Bayes. arXiv [stat.ML] (2013).

735 30. Türei, D. et al. Integrated intra- and intercellular signaling knowledge for multicellular omics analysis.

736 doi:10.1101/2020.08.03.221242.

737 31. Mclnnes, L., Healy, J. \& Melville, J. UMAP: Uniform Manifold Approximation and Projection for Dimension

738 Reduction. arXiv [stat.ML] (2018). 